\title{
Toll-like receptors of deuterostome invertebrates
}

\author{
Honoo Satake* and Toshio Sekiguchi \\ Bioorganic Research Institute, Suntory Foundation for Life Sciences, Osaka, Japan
}

\section{Edited by:}

Larry J. Dishaw, University of South

Florida, USA

\section{Reviewed by:}

Dmitri V. Krysko, Ghent University, Belgium

Shun-Ichiro Kawabata, Kyushu

University, Japan

Jared C. Roach, Institute for Systems

Biology, USA

\section{*Correspondence:}

Honoo Satake, Bioorganic Research Institute, Suntory Foundation for Life Sciences, 1-1-1 Wakayamadai,

Shimamoto, Mishima, Osaka 618-8503, Japan.

e-mail: satake@sunbor.or.jp
Defensive systems against pathogens are responsible not only for survival or lifetime of an individual but also for the evolution of a species. Innate immunity is expected to be more important for invertebrates than mammals, given that adaptive immunity has not been acquired in the former. Toll-like receptors (TLRs) have been shown to play a crucial role in host defense of pathogenic microbes in innate immunity of mammals. Recent genome-wide analyses have suggested that TLR or their related genes are conserved in invertebrates. In particular, numerous TLR-related gene candidates were detected in deuterostome invertebrates, including a sea urchin (222 TLR-related gene candidates) and amphioxus (72TLR-related gene candidates). Molecular phylogenetic analysis verified that most of sea urchin or amphioxus TLR candidates are paralogous, suggesting that these organisms expanded TLR-related genes in a species-specific manner. In contrast, another deuterostome invertebrate, the ascidian Ciona intestinalis, was found to possess only two TLR genes. Moreover, CionaTLRs, Ci-TLR1 and Ci-TLR2, were shown to possess "hybrid" functionality of mammalian TLRs. Such functionality of Ci-TLRs could not be predicted by sequence comparison with vertebrate TLRs, indicating confounding evolutionary lineages of deuterostome invertebrate TLRs or their candidates. In this review article, we present recent advances in studies of TLRs or their candidates among deuterostome invertebrates, and provide insight into an evolutionary process of TLRs.

\section{Keywords: Toll-like receptor, innate immunity, evolution, deuterostome invertebrate, diversity}

\section{INTRODUCTION}

Innate immunity is a primary defense system against invading pathogens in invertebrates, in which adaptive immune systems have not been fully developed. Invertebrate innate immunity consists of diverse pathogen recognition systems including: hemolymph coagulation, lectin-mediated complement activation, antimicrobial peptides, and variable region-containing chitinbinding proteins (Nonaka, 2001; Fujita, 2002; Khalturin et al., 2004; Iwanaga and Lee, 2005; Miller et al., 2007; Rast and MessierSolek, 2008; Bonura et al., 2009; Nonaka and Satake, 2010; Satake and Sasaki, 2010; Dishaw et al., 2011). Most invertebrate immune systems diverged among species, whereas recent genome surveys of various organisms revealed that Toll-like receptors (TLRs) or their related proteins are essentially conserved in an extensive range of organisms from cnidarians to mammals. In particular, deuterostome invertebrates and vertebrates conserve TLR-directed innate immunity (Akira and Takeda, 2004; Khalturin et al., 2004; Dunne and O'Neill, 2005; Iwanaga and Lee, 2005; Roach et al., 2005; Takeda and Akira, 2005; Ishii et al., 2007; Takano et al., 2007; Davidson et al., 2008; Fedders et al., 2008; Matsuo et al., 2008; Oshiumi et al., 2008; Rast and Messier-Solek, 2008; Bosch et al., 2009; Schikorski et al., 2009; Yu et al., 2009; Macagno et al., 2010; Satake and Sasaki, 2010; Cuvillier-Hot et al., 2011; Park et al., 2011; Wu et al., 2011). These studies not only suggest that the TLR system of immune receptors is the most widely distributed throughout the deuterostomes but also reveal several characteristics of TLRs specific to deuterostome invertebrates.

Deuterostomia consists of six major phyla: Xenoturbellida, Echinodermata, Hemichordata, Cephalochordata, Urochordata, and Vertebrata. Each phylum shares the basal developmental process in which the blastopore is converted into the mouth, although their forms, lifestyles, and life spans are dramatically different within deuterostome invertebrates, making them attractive targets for the molecular and functional evolution of various biological phenotypes and systems, including innate immunity. To date, the genomes of three deuterostome invertebrates, Ciona intestinalis (ascidian, Urochordata), Branchiostoma floridae (amphioxus, Cephalochordata), and Strongylocentrotus purpuratus (sea urchin, Echinodermata) have been determined. Genomic analyses have verified that sea urchin, amphioxus, and ascidian possess 222, 72, and 2 ascidian TLR candidates (Hibino et al., 2006; Rast et al., 2006; Huang et al., 2008; Sasaki et al., 2009), indicating high diversity in the number of TLR candidates among deuterostome invertebrates. In contrast, the number of TLRs in vertebrates does not dramatically differ among species; 10 for human and 12 for Takifugu (Roach et al., 2005; Matsuo et al., 2008). These findings suggest that molecular and functional studies of deuterostome invertebrate TLRs have led to their evolution and diversity as well as to the elucidation of molecular mechanisms underlying their innate immunity. In this review, we provide an overview and possible evolutionary scenarios of deuterostome invertebrate TLRs.

\section{TLRS: CHARACTERIZATION, FAMILY MEMBERS, PATHOGEN RECOGNITION, AND SIGNAL TRANSDUCTION}

Toll-like receptors were characterized originally as a mammalian ortholog of the Drosophila melanogaster (fruitfly) transmembrane protein Toll, which is responsible for antifungal protection and 
Table 1 | Comparison of human and Ciona TLRs.

\begin{tabular}{|c|c|c|c|}
\hline TLR & Number of LRR & Ligands & $\begin{array}{l}\text { Intracellular } \\
\text { localization }\end{array}$ \\
\hline hTLR1 (with TLR2) & 5 & Triacylated lipoprotein & Plasma membrane \\
\hline hTLR2 & 9 & $\begin{array}{l}\text { Zymosan (yeast cell wall) } \\
\text { 1,3- } \beta \text {-glucan lipoarabinomannan } \\
\text { Heat-killed Legionella pneumophila (HKLP, Gram-negative) } \\
\text { Heat-killed Staphylococcu aureus (HKSA, Gram-positive) } \\
\text { Glycosylphosphatidylinositol (GPI)-anchored glycoprotein }\end{array}$ & Plasma membrane \\
\hline hTLR3 & 17 & Poly(I:C) (double-stranded RNA) & Endosome \\
\hline hTLR4 (with MD2) & 11 & $\begin{array}{l}\text { LPS (lipopolysaccharide from Gram-negative bacteria) } \\
\text { Lipid A (lipid component of LPS) }\end{array}$ & Plasma membrane \\
\hline hTLR5 & 10 & Flagellin (bacterial flagellar filament) & Plasma membrane \\
\hline hTLR6 & 6 & $\begin{array}{l}\text { MALP-2 (mycoplasma-derived macrophage-activating lipopeptide) } \\
\text { FSL1 (mycoplasma-derived lipoprotein) }\end{array}$ & Plasma membrane \\
\hline hTLR7 & 14 & Imidazoquimod (imidazoquinolone amino acid analog) & Endosome \\
\hline hTLR8 & 16 & Single-stranded RNA & Endosome \\
\hline hTLR9 & 19 & Unmethylated CpG DNA & Endosome \\
\hline Ci-TLR1 & 7 & $\begin{array}{l}\text { Zymosan (yeast cell wall), heat-killed Legionella pneumophila (HKLP, } \\
\text { Gram-negative), poly(l:C) (double-stranded RNA), flagellin (bacterial flagellar } \\
\text { filament) }\end{array}$ & $\begin{array}{l}\text { Plasma membrane } \\
\text { and endosome }\end{array}$ \\
\hline Ci-TLR2 & 13 & & \\
\hline
\end{tabular}

determination of the dorsal/ventral pattern (Lemaitre et al., 1996; Hoffmann, 2003). TLRs are type I transmembrane proteins, featured by an intracellular Toll/Interleukin-1 receptor (TIR) domain and leucine-rich repeat (LRR) motifs in the extracellular domain (Akira and Takeda, 2004; Dunne and O'Neill, 2005; Takeda and Akira, 2005), and have been found to play pivotal roles in mammalian host defenses via the innate immune system. LRRs exhibit specific pathogenic ligand recognition, and TIRs participate in activation of signaling pathways. TLR4 is the first mammalian TLR that was described and was characterized by functional analyses of a mouse deficient in host defense against a typical endotoxin, lipopolysaccharide (LPS; Poltorak et al., 1998). Other TLRs were structurally and functionally characterized in human and mice. To date, 10 functional human TLRs (hTLRs) have been identified (Akira and Takeda, 2004; Dunne and O'Neill, 2005; Roach et al., 2005; Takeda and Akira, 2005; Park et al., 2011). As summarized in Table 1, each hTLR differs in the number and organization of LRRs, and recognizes their specific ligands, namely pathogenassociated molecular patterns (PAMPs). The diversity in the numbers and organization of LRR domains are believed to enable the specific and sensitive recognition of PAMPs by respective TLRs (Akira and Takeda, 2004; Dunne and O'Neill, 2005; Takeda and Akira, 2005). TLRs are expressed in the lung, kidney, small intestine, stomach, ovary, and testis as well as in immune cells such as lymphocytes, macrophages, and dendritic cells (Akira and Takeda, 2004; Dunne and O'Neill, 2005; Takeda and Akira, 2005). TLR1, TLR2, TLR4, TLR5, and TLR6 are responsible for recognition of extracellular microbial pathogenic components on plasma membranes, whereas TLR3, TLR7, TLR8, and TLR9, exclusively present on endosomes, participate in defensive responses to viral DNA or RNA incorporated into the cytoplasm (Akira and Takeda, 2004; Dunne and O'Neill, 2005; Takeda and Akira, 2005). Thus, TLRs are categorized into plasma membrane TLR groups (TLR1, -2, $-4,-5$, and -6 ) or endosome TLR groups (TLR3, $-7,-8$, and -9 ) in terms of their intracellular localization. In addition, TLR4 requires an extracellular associated protein, MD2, to recognize LPS (Shimazu et al., 1999; Akashi et al., 2000; Dunne and O'Neill, 2005; Takeda and Akira, 2005). Moreover, the tertiary structures of several TLR-ligand complexes have been resolved (Jin and Lee, 2008; Liu et al., 2008; Park et al., 2009). Interaction of TLRs with their specific PAMPs triggers signal transduction pathways via adaptor proteins (MyD88, TIRAP, TRIF, and TRAM) followed by activation of a wide range of inducible transcriptional factors such as: NF- $\mathrm{B}, \mathrm{AP}-1$, and IRF, leading to production of inflammatory cytokines, chemokines, and/or type I interferon (Akira and Takeda, 2004; Dunne and O'Neill, 2005; Takeda and Akira, 2005). As stated above, molecular forms and biological roles of TLR were investigated extensively in mammals. However, over the past decades, deuterostome invertebrates also have been shown to share, at least in part, the TLR-triggered innate immune system.

\section{TLR IN CIONA INTESTINALIS: THE FIRST FUNCTIONALLY CHARACTERIZED DEUTEROSTOME INVERTEBRATE TLRS}

A Ciona genome survey and molecular cloning revealed the presence of two TLRs in C. intestinalis, Ci-TLR1 and Ci-TRL2 (Azumi et al., 2003; Sasaki et al., 2009), both of which are composed of a TIR, a transmembrane, and a LRR domain. Moreover, 7 and 13 LRRs are found in Ci-TLR1 and Ci-TLR2, respectively (Figure 1). The $c i$-tlr1 and $c i$-tlr 2 genes are expressed intensively in the stomach, intestine, and numerous hemocytes and, to a lesser degree, the central nervous system (Sasaki et al., 2009). These findings indicate that Ci-TLRs function mainly in the alimentary tracts and hemocytes. Interestingly, both Ci-TLRs, unlike any vertebrate TLRs, are present on the plasma membrane and a number of late 


\section{C.intestinalis}

Human
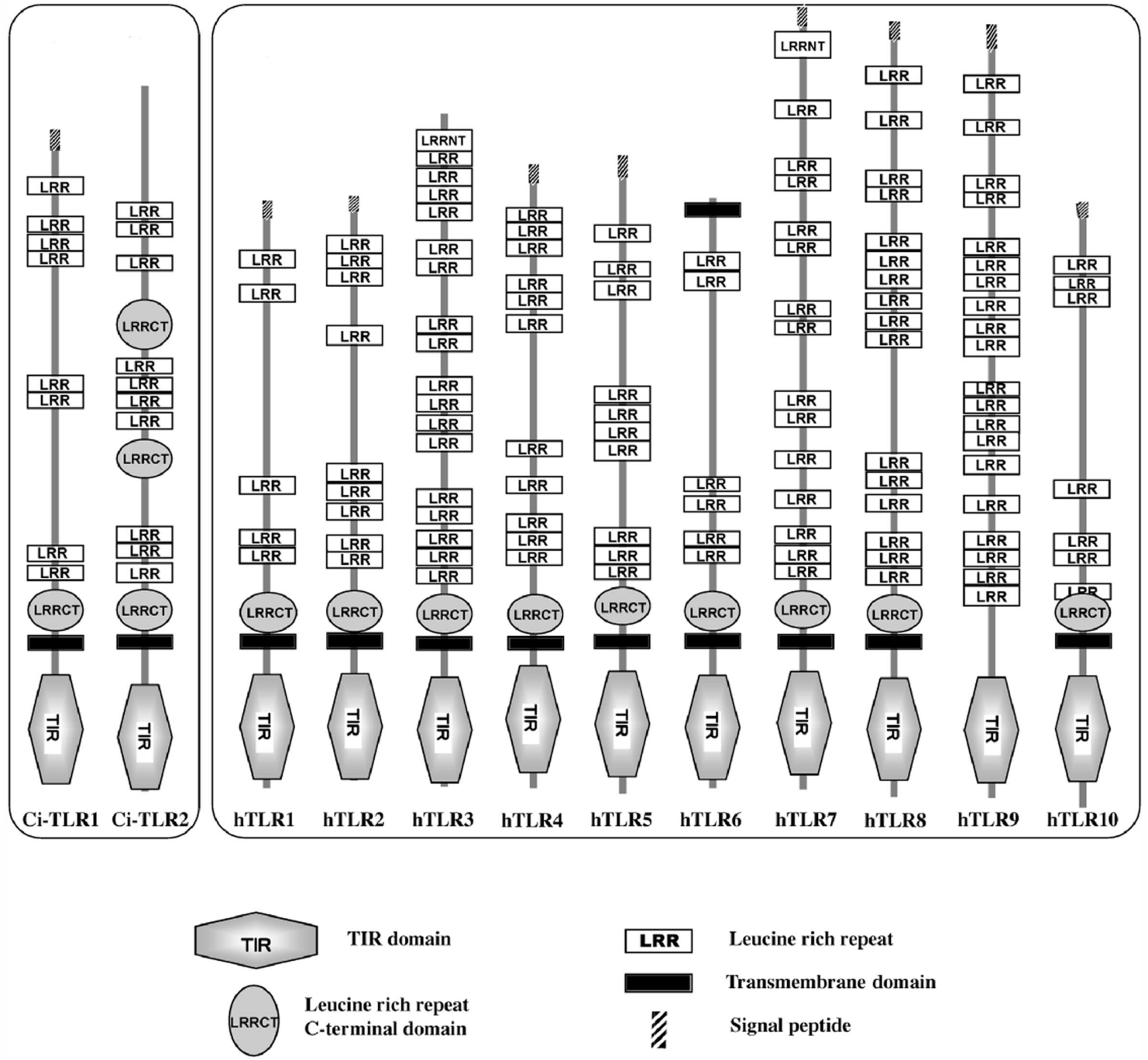

FIGURE 1 | Structural organization of Ciona TLRs (Ci-TLR) and human TLRs (hTLR)

endosomes (Sasaki et al., 2009). A striking feature is that Ci-TLR1 and Ci-TLR2 expressed in HEK293MSR cells activated NF- $\mathrm{B}$ in response to multiple TLR ligands (Table 1 ), which are recognized by different mammalian TLRs: zymosan (Saccharomyces cerevisiae cell wall) for hTLR2, heat-killed Legionella pneumophila (HKLP, a Gram-negative bacterium) for hTLR2, double-stranded RNA poly(I:C) for hTLR3, and Salmonella typhimurium Flagellin (the major component of the bacterial flagellar filament) for hTLR5, which elicited a dose-dependent transactivation of NF$\kappa \mathrm{B}$ in the ci-tlr1- or ci-tlr2-expressing cells (Sasaki et al., 2009). Poly(I:C) and flagellin also elicited approximately 4- and 10-fold $\mathrm{Ci}-\mathrm{TNF} \alpha$ expression in the stomach, anterior and/or middle intestine where $c i$-tlr1 or ci-tlr2 are abundantly expressed, but not in the posterior intestine where expression of ci-tlrs was not detected (Sasaki et al., 2009). These data lead to two important conclusions. First, Ci-TLRs, like vertebrate TLRs, directly recognize PAMPs and trigger the transactivation of NF- $\kappa \mathrm{B}$. This is in contrast with Drosophila Toll, which requires insect-specific accessory proteins for pathogen recognition and the following signal transduction (Hoffmann, 2003). Consequently, Ci-TLRs share the essential ligand recognition and signaling mechanisms with the vertebrate TLR family, which is compatible with a previous genomic survey clarifying the presence of Ciona orthologs of TLR-signaling factors such as MyD88, NF- $\kappa$ B, and TNF $\alpha$ (Azumi et al., 2003). Second, Ci-TLRs recognize more extensive PAMPs than vertebrate TLRs (Table 1). This is the first report on TLRs that respond to multiple PAMPs of respective hTLRs. The PAMP recognition by Ci-TLRs is in good agreement with intracellular localization to both the plasma membrane and endosome, given that poly(I:C) is recognized by hTLR3 present on endosome, and that hTLR2 and hTLR5 on the plasma membrane interact with zymosan, HKLP, and flagellin. Collectively these 
multiple PAMP recognition specificities and intracellular localization differences provide evidence that Ci-TLRs are functionally hybrid TLRs of vertebrate cell-surface TLRs (hTLR1, -2, -4, $5,-6$ ) and endosome TLRs (hTLR3, -7, -8, -9). Such functions of Ci-TLRs are not predicted on the basis of sequence homology to hTLRs, leading to the unique location in the molecular phylogenetic trees of chordate TLRs and their candidates. It is noteworthy that both Ci-TLRs elicited equipotent NF- $\kappa \mathrm{B}$ activation in response to the same ligands, whereas Ci-TLRs display different structural organization of LRRs (Table 1). These findings suggest that recognition of PAMPs and that the resulting signaling by deuterostome invertebrate TLRs (including Ci-TLRs) cannot be elucidated or even predicted by sequence homology-based analyses.

Ci-TLRs are devoid of any responses to LPS (Sasaki et al., 2009). In C. intestinalis, in vivo challenge of LPS resulted in the upregulation of a TLR-triggering cytokine, TNF $\alpha$ (Parrinello et al., 2008), and a mannose-binding lectin-like collectin (Bonura et al., 2009), indicating that C. intestinalis possesses a LPS-responsive system. In mammals, LPS is recognized by a complex of hTLR4 and MD2, not by hTLR4 alone (Shimazu et al., 1999; Akashi et al., 2000; Dunne and O'Neill, 2005; Takeda and Akira, 2005). This is consistent with the data showing that Ci-TLRs fail to recognize LPS. Moreover, no MD2 homolog was detected in the $C$. intestinalis genome (Azumi et al., 2003). Taken together, these findings lead to two hypotheses: (1) Ci-TLRs possess no ability to recognize LPS, and (2) other LPS-responsive TLR systems are functional in C. intestinalis. Interestingly, recognition of LPS by non-TLR receptors in fish is also suggested (Iliev et al., 2005), i.e., recognition of LPS by TLR4 with MD2 may have been acquired during the evolution of vertebrates. Alternatively, Ci-TLRs may respond to LPS through formation of a complex with other associated proteins than MD2. If this is true, the question is raised as to whether such an MD2-independent LPS-responsive system is specific to $C$. intestinalis or is common among deuterostome invertebrates. Both molecular and functional studies will address this issue.

Ci-TLRs display moderate differences in their expression despite almost identical ligand recognition; Ci-TLR1 is expressed much more in the anterior intestine, compared to Ci-TLR2 (Sasaki et al., 2009). On the other hand, Ci-TLR1 and Ci-TLR2 share their PAMPs (Table 1) and exhibit equipotent NF- $\kappa \mathrm{B}$ activation. These findings indicate the possibility that Ci-TLR1 and Ci-TLR2 have different immunological and/or biological roles. For instance, they may differentially recognize other PAMPs that have not been investigated or may have another signaling cascade that differs from NF- $\kappa \mathrm{B}$ activation. Alternatively, Ci-TLR1 and Ci-TLR2 are anticipated to play a differential role in other biological functions other than innate immunity. Recently, TLRs have also been shown to recognize several high-concentration endogenous ligands, including heme and endogenous double-stranded RNA resulting from apoptosis (Figueiredo et al., 2007; Wu et al., 2008). These findings suggest biological roles for TLRs as a sensor of unusual generation of endogenous components, and that the expression profiles for Ci-TLRs reflect different functions in recognition of endogenous ligands. Elucidation of their principal functional roles is underway in our laboratory.

\section{AMPHIOXUS TLRs}

Seventy-two TLR or TLR-related genes were detected in the genome of B. floridae (Huang et al., 2008). Recently, an amphioxus TLR (bbtTLR) was identified in another amphioxus species, Branchiostoma belcheri tsingtauense (Yuan et al., 2009). The expression of bbttlr was initiated at the late blastula/early gastrula stage, and observed from the embryonic to larval stages (Yuan et al., 2009), suggesting a developmental role. In adults, the bbttlr expression was detected predominantly in the gill, skin, and gut, and, to a lesser degree in the ovaries, muscles, hemocytes, and notochord (Yuan et al., 2009). Such bbttlr expression also suggests an immunological function in the innate immunity, given that the gill, skin, and gut are exposed directly to pathogens. Interestingly, LPS, lipoteichoic acids, and Gram-negative bacteria (Vibrio vulnificus) upregulate the bbtTLR transcript, whereas poly(I:C) and glucan downregulate the transcript (Yuan et al., 2009). These data support the hypothesis that bbtTLR participates in defense responses to certain pathogens. However, neither intact nor chimeric bbtTLR recognize LPS, lipoteichoic acids, peptidoglycan, poly(I:C), or glucan, although bbtTLR was localized to the plasma membrane (Yuan et al., 2009). This failure is due to the heterologous expression of bbtTLR in human culture cells. Otherwise, other TLR ligands are likely to activate bbtTLR, which may require a certain associated protein for recognition of ligands, as MD2 is a prerequisite for recognition of LPS by hTLR4 (Shimazu et al., 1999; Akashi et al., 2000; Dunne and O'Neill, 2005; Takeda and Akira, 2005). Elucidation of function of bbtTLR awaits further analysis.

\section{SEA URCHIN TLRS: THE LARGEST REPERTOIRE}

The genome-wide analysis of the purple sea urchin, Strongylocentrotus purpuratus, detected 222 TLR-like genes (Hibino et al., 2006; Rast et al., 2006). A molecular phylogenetic tree analysis led to classification of $S$. purpuratus TLR genes to seven major groups (Hibino et al., 2006). The vast majority of sea urchin TLR genes display higher similarity to each other than to TLRs of other species TLRs and are encoded in tandem arrays, suggesting an enormous gene expansion that is specific to Strongylocentrotus TLRs (Hibino et al., 2006). Although tissue distribution of sea urchin TLRs has yet to be investigated, TLR cDNAs were identified from coelomocyte. Major classes of sea urchin TLR cDNAs were not expressed in embryos prior to the end of gastrulation. These data support the hypothesis that sea urchin TLRs have primarily an immunological role, unlike insect Toll. Function and cellular localization of sea urchin TLRs await further investigation. Random investigations of all 222 sea urchin TLRs is difficult. Nevertheless, TLR-signalingassociated protein homologs such as: MyD88, NF- $\mathrm{B}$, and TNF $\alpha$, were detected in the genome of S. purpuratus (Hibino et al., 2006), implying that the essential TLR-triggered innate immune system is conserved in sea urchin. Thus, a promising target is several sea urchin TLRs that are abundantly expressed in immune tissues and cells, such as alimentary tract or hemocytes.

\section{EVOLUTIONARY SCENARIO OF TLR IN DEUTEROSTOME INVERTEBRATES}

Amphioxus and sea urchin possess a great number of TLRs or related genes (Hibino et al., 2006; Rast et al., 2006; Huang et al., 2008). Furthermore, molecular phylogenetic analyses demonstrate 


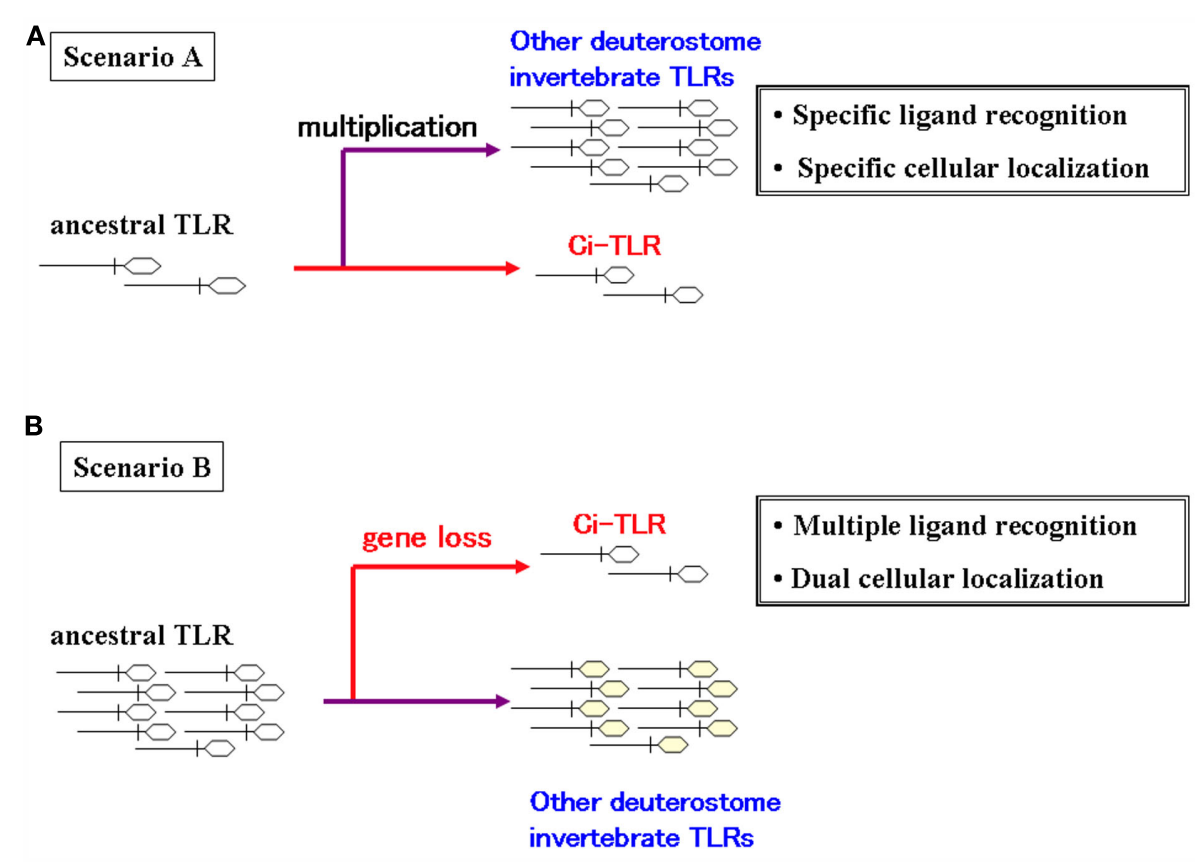

FIGURE 2 | Possible evolutionary scenarios of deuterostome invertebrate TLRs. (A) only a few TLRs or their related genes might have existed in a common deuterostome ancestor or (B) a common deuterostome ancestor might have numerous TLR family genes.

that most of these genes are generated via species-specific gene duplication, suggesting that these deuterostome invertebrates expand TLRs or their related genes in unique lineages of innate immunity, if most of the genes are functional (Hibino et al., 2006; Huang et al., 2008; Rast and Messier-Solek, 2008). In contrast, C. intestinalis possesses only two authentic TLRs (Sasaki et al., 2009), leading to two evolutionary scenarios of TLRs or their related genes (Figure 2). First, only a few TLR or their related genes might have existed in a common deuterostome ancestor. In this case, $C$. intestinalis conserves the ancestral characteristics, whereas sea urchins and amphioxus expanded their TLR or related gene paralogs during their divergence in concert with the variation of their lifetimes, life cycles, or environments. This presumption is also compatible with the aforementioned molecular phylogenetic analyses of sea urchin and amphioxus TLRs and their related genes. Moreover, the ancestral TLRs, as seen in Ci-TLRs, might have responded to multiple PAMPs, which are differentially recognized by respective currently existing vertebrate TLRs and have been present both on cell surface and on endosomes. In keeping with this, vertebrate and probably most of sea urchins and amphioxus TLRs might have acquired their specific PAMP recognition and intracellular localization during their evolution. Alternatively, a common deuterostome antecedent might have numerous TLR family genes, as seen in sea urchin and amphioxus. If true, $C$. intestinalis should have lost a large part of ancestral TLR family genes, instead, Ci-TLRs are highly likely to have acquired multiple PAMP recognition and intracellular localization. Therefore, characterization of PAMPs and intracellular localization of sea urchin and amphioxus TLRs is expected to contribute not only to elucidation of their biological roles but also to investigation of molecular and functional divergence of the deuterostome TLR family.
It is noteworthy that all deuterostome invertebrates are marine organisms that should establish an immune system specific to ocean pathogens, given that these pathogens do not completely coincide with those for land mammals. This view may be supported by difference in PAMP recognition between Ci-TLRs and hTLR2; two ligands for TLR2, zymosan and HKLP exhibit prominent activation of Ci-TLRs, whereas other hTLR2 ligands, including HKLM, 1,3- $\beta$-glucan, and HKSA, are devoid of any activity at Ci-TLRs (Sasaki et al., 2009). Collectively, these findings indicate the immunological significance of the considerably high diversity in sequences and organization of LRRs, which is consistent with the recognized PAMPs. Innate immunity against pathogens is expected to be closely related to the lifetimes and life cycles of organisms. For instance, the sea urchin has an extremely long lifetime (30-40 years), indicating that this organism is endowed with excellent defense against pathogens. In contrast, the lifetime of $C$. intestinalis is only 3 months. Such differences imply that the numbers of TLRs or TLR-like candidate genes may be correlate with lifespans of organisms. In keeping with this, the molecular and functional characterization of TLRs of another ascidian, Halocynthia roretzi, should lead to incisive findings due to its much longer lifespan (up to 8 years) and its distribution in relatively different habitats. Feeding strategies may also participate in the diversity in the number and functions of TLRs. For instance, Ciona feeds exclusively on plant planktons and sea urchin feeds on both dead fish and plant planktons. These feeding differences suggest that sea urchin may encounter more various pathogens than Ciona (Davidson et al., 2008). Intriguingly, a land annelid, the blood-sucking leech Helobdella robusta, was found to possess 16 TLR-like genes, whereas the marine non-blood-sucking annelid Capitella capitata was found to possess 105 TLR-like genes. The 
authors hypothesized that such low number of $H$. robusta TLRlike genes compared with that of $C$. capitata may be due to the possibility that blood-sucking leeches obtain functional immune components from their host animals.

\section{CONCLUSIONS AND PERSPECTIVES}

Deuterostome TLRs or their candidate genes have been identified with growing efficiency over the past few years. However, Ci-TLRs are at present the only functionally characterized TLRs of deuterostome invertebrates. This can be attributed to the high numbers of sea urchin and amphioxus TLRs, high molecular divergence in the numbers and organization of the LRRs among deuterostome invertebrate TLR (or TLR-like) genes, and severe limitations of homology-based sequence analysis for characterizing crucial functions of PAMPs, cellular localization, and signaling pathways, which are of interest in amphioxus and sea urchin. Apart from the high number of sea urchin and amphioxus TLR candidate genes, one of the difficulties in functional analyses of deuterostome invertebrate TLRs results from failure of functional expression of the TLRs in certain heterologous host cultured cells due to the lack of availability of homologous expression systems including cognate cultured cell lines, as seen in studies of amphioxus and cyclostomes (Ishii et al., 2007; Yuan et al.,

\section{REFERENCES}

Akashi, S., Shimazu, R., Ogata, H., Nagai, Y., Takeda, K., Kimoto, M., and Miyake, K. (2000). Cutting edge: cell surface expression and lipopolysaccharide signaling via the toll-like receptor 4-MD-2 complex on mouse peritoneal macrophages. J. Immunol. 164, 3471-3475.

Akira, S., and Takeda, K. (2004). Tolllike receptor signalling. Nat. Rev. Immunol. 4, 499-511.

Azumi, K., De Santis, R., De Tomaso, A., Rigoutsos, I., Yoshizaki, F., Pinto, M. R., Marino, R., Shida, K., Ikeda, M., Ikeda, M., Arai, M., Inoue, Y., Shimizu, T., Satoh, N., Rokhsar, D. S., Du Pasquier, L., Kasahara, M., Satake, M., and Nonaka, M. (2003). Genomic analysis of immunity in a urochordate and the emergence of the vertebrate immune system: "waiting for Godot." Immunogenetics 55, 570-581.

Bonura, A., Vizzini, A., Salerno, G., Parrinello, N., Longo, V., and Colombo, P. (2009). Isolation and expression of a novel MBL-like collectin cDNA enhanced by LPS injection in the body wall of the ascidian Ciona intestinalis. Mol. Immunol. 46, 2389-2394.

Bosch, T. C. G., Augustin, R., AntonErxleben, F., Fraune, S., Hemmrich, G., Zill, H., Rosenstiel, P., Jacobs, G., Schreiber, S., Leippe, M., Stanisak, M., Grötzinger, J., Jung, S., Podschun, R., Bartels, J., Harder, J., and Schröder, J. M. (2009). Uncovering the evolutionary history of innate immunity: the simple metazoan Hydra uses epithelial cells for host defense. Dev. Comp. Immunol. 33, 559-569.

Cuvillier-Hot, V., Boidin-Wichlacz, C., Slomianny, C., Salzet, M., and Tasiemski, A. (2011). Characterization and immune function of two intracellular sensors, HmTLR1 and HmNLR, in the injured CNS of an invertebrate. Dev. Comp. Immunol. 35, 214-226.

Davidson, C. R., Best, N. M., Francis, J. W., Cooper, E. L., and Wood, T. C. (2008). Toll-like receptor genes (TLRs) from Capitella capitata and Helobdella robusta (Annelida). Dev. Comp. Immunol. 32, 608-612.

Dishaw, L. J., Giacomelli, S., Melillo, D. Zucchetti, I., Haire, R. N., Natale, L., Russo, N. A., De Santis, R., Litman, G. W., and Pinto, M. R. (2011). A role for variable regioncontaining chitin-binding proteins (VCBPs) in host gut-bacteria interactions. Proc. Natl. Acad. Sci. U.S.A. 108, 16747-16752.

Dunne, A., and O'Neill, L. A. (2005). Adaptor usage and Toll-like receptor signaling specificity. FEBS Lett. 579, 3330-3335.

Fedders, H., Michalek, M., Grötzinger, J., and Leippe, M. (2008). An exceptional salt-tolerant antimicrobial peptide derived from a novel gene family of haemocytes of the marine invertebrate Ciona intestinalis. Biochem. J. 416, 65-75.

Figueiredo, R. T., Fernandez, P. L., Mourao-Sa, D. S., Porto, B. N.,

2009). Therefore, establishment of cultured cells derived from each organism will strongly enhance various functional studies of deuterostome invertebrate TLRs. In this context, Ci-TLRs possess distinct advantages as they can be expressed efficiently in HEK293MSR cells, thus facilitating the characterization of their PAMPs, intracellular localization, and signaling.

Also of biological and immunological significance is the characterization of functional relationship TLRs and other innate immune systems, including the complement activation system, antibacterial peptides synthesis and hemolymph coagulation. For instance, it has been speculated that TLR-triggered signaling in $C$. intestinalis can lead to complement activation. Furthermore, variable region-containing chitin-binding proteins may serve cooperatively as mediators of immunity along with TLRs. Integration of functional roles is expected to pave the way to the characterization of innate immunity at the molecular level in deuterostome invertebrates.

\section{ACKNOWLEDGMENTS}

We thank Ms. Kazuko Hirayama and all members of Maizuru Fisheries Research Station for cultivation of the ascidians. All ascidians were provided by Kyoto University through the National Bio-Resource Project of the MEXT, Japan.

Dutra, F. F., Alves, L. S., Oliveira, M. F., Oliveira, P. L., Graça-Souza, A. V., and Bozza, M. T. (2007). Characterization of heme as activator of Toll-like receptor 4. J. Biol. Chem. 282, 20221-20229.

Fujita, T. (2002). Evolution of the lectin-complement pathway and its role in innate immunity. Nat. Rev. Immunol. 2, 346-353.

Hibino, T., Loza-Coll, M., Messier, C., Majeske, A. J., Cohen, A. H., Terwilliger, D. P., Buckley, K. M., Brockton, V., Nair, S. V., Berney, K., Fugmann, S. D., Anderson, M. K., Pancer, Z., Cameron, R. A., Smith, L. C., and Rast, J. P. (2006). The immune gene repertoire encoded in the purple sea urchin genome. Dev. Biol. 300, 349-365.

Hoffmann, J. A. (2003). The immune response of Drosophila. Nature 426, 33-38.

Huang, S., Yuan, S., Guo, L., Yu, Y., Li, J., Wu, T., Liu, T., Yang, M., Wu, K., Liu, H., Ge, J., Yu, Y., Huang, H., Dong, M., Yu, C., Chen, S., and $\mathrm{Xu}, \mathrm{A}$. (2008). Genomic analysis of the immune gene repertoire of amphioxus reveals extraordinary innate complexity and diversity. Genome Res. 18, 1112-1126.

Iliev, D. B., Roach, J. C., Mackenzie, S., Planas, J. V., and Goetz, F. W. (2005). Endotoxin recognition: in fish or not in fish? FEBS Lett. 579, 6519-6528.

Ishii, A., Matsuo, A., Sawa, H., Tsujita, T., Shida, K., Matsumoto, M., and Seya, T. (2007). Lamprey TLRs with properties distinct from those of the variable lymphocyte receptors. $J$. Immunol. 178, 397-406.

Iwanaga, S., and Lee, B. L. (2005). Recent advances in the innate immunity of invertebrate animals. J. Biochem. Mol. Biol. 38, 128-150.

Jin, M. S., and Lee, J. O. (2008). Structures of the toll-like receptor family and its ligand complexes. Immunity 29, 182-191.

Khalturin, K., Panzer, Z., Cooper, M. D., and Bosch, T. C. G. (2004). Recognition strategies in the innate immune system of ancestral chordates. Mol. Immunol. 41, 1077-1187.

Lemaitre, B., Nicolas, E., Michaut, L., Reichhart, J. M., and Hoffmann, J. A. (1996). The dorsoventral regulatory gene cassette spätzle/Toll/cactus controls the potent antifungal response in Drosophila adults. Cell 86, 973-983.

Liu, L., Botos, I., Wang, Y., Leonard, J. N., Shiloach, J., Segal, D. M., and Davies, D. R. (2008). Structural basis of toll-like receptor 3 signaling with double-stranded RNA. Science 320, 379-381.

Macagno, E. R., Gaasterland, T., Edsall, L., Bafna, V., Soares, M. B., Scheetz, T., Casavant, T., Da Silva, C., Wincker, P., Tasiemski, A., and Salzet, M. (2010). Construction of a medicinal leech transcriptome database and its application to the identification of leech homologs of neural and innate immune genes. BMC Genomics 11, 407. doi:10.1186/1471-2164-11-407 
Matsuo, A., Oshiumi, H., Tsujita, T., Mitani, H., Kasai, H., Yoshimizu, M., Matsumoto, M., and Seya, T. (2008). Teleost TLR22 recognizes RNA duplex to induce IFN and protect cells from birnaviruses. J. Immunol. 18, 3474-3485.

Miller, D. J., Hemmrich, G., Ball, E. E., Hayward, D. C., Khalturin, K., Funayama, N., Agata, K., and Bosch, T. C. G. (2007). The innate immune repertoire in cnidaria - ancestral complexity and stochastic gene loss. Genome Biol. 8, R59.

Nonaka, M. (2001). Evolution of the complement system. Curr. Opin. Immunol. 13, 69-73.

Nonaka, M., and Satake, H. (2010). Urochordate immunity. Adv. Exp. Med. Biol. 708, 302-310.

Oshiumi, H., Matsuo, A., Matsumoto, M., and Seya, T. (2008). Panvertebrate Toll-like receptors during evolution. Curr. Genomics 9, 488-493.

Park, B. S., Song, D. H., Kim, H. M., Choi, B. S., Lee, H., and Lee, J. O. (2009). The structural basis of lipopolysaccharide recognition by the TLR4-MD-2 complex. Nature 458, 1191-1195.

Park, H. J., Hahn, W. H., Suh, J. S., Kim, M. J., Kang, S. W., Lee, J. S., Kim, J. W., Chung, J. H., and Cho, B. S. (2011). Association between toll-like receptor 10 (TLR10) gene polymorphisms and childhood IgA nephropathy. Eur. J. Pediatr. 170, 503-509.

Parrinello, N., Vizzini, A., Arizza, V., Salerno, G., Parrinello, D.,
Cammarata, M., Giaramita, F. T., and Vazzana, M. (2008). Enhanced expression of a cloned and sequenced Ciona intestinalis TNF $\alpha$-like $(\mathrm{CiTNF} \alpha)$ gene during the LPS-induced inflammatory response. Cell Tissue Res. 334, 305-317.

Poltorak, A., He, X., Smirnova, I., Liu, M. Y., Van Huffel, C., Du, X., Birdwell, D., Alejos, E., Silva, M., Galanos, C., Freudenberg, M., Ricciardi-Castagnoli, P., Layton, B., and Beutler, B. (1998). Defective LPS signaling in $\mathrm{C} 3 \mathrm{H} / \mathrm{HeJ}$ and $\mathrm{C} 57 \mathrm{BL} / 10 \mathrm{ScCr}$ mice: mutations in Tlr4 gene. Science 282, 2085-2088.

Rast, J. P., and Messier-Solek, C. (2008). Marine invertebrate genome sequences and our evolving understanding of animal immunity. Biol. Bull. 214, 274-283.

Rast, J. P., Smith, L. C., Loza-Coll, M., Hibino, T., and Litman, G. W. (2006). Genomic insights into the immune system of the sea urchin. Science 314, 952-956.

Roach, J. C., Glusman, G., Rowen, L., Kaur, A., Purcell, M. K., Smith, K. D., Hood, L. E., and Aderem, A. (2005). The evolution of vertebrate Toll-like receptors. Proc. Natl. Acad. Sci. U.S.A. 102, 9577-9582.

Sasaki, N., Ogasawara, M., Sekiguchi, T., Kusumoto, S., and Satake, H. (2009). Toll-like receptors of the ascidian, Ciona intestinalis: prototypes with hybrid functionalities of vertebrate Toll-like receptors. J. Biol. Chem. 284, 2733627343.
Satake, H., and Sasaki, N. (2010). Comparative view on Toll-like receptors of lower animals. Zool. Sci. 27, 154-161.

Schikorski, D., Cuvillier-Hot, V., BoidinWichlacz, C., Slomianny, C., Salzet, M., and Tasiemski, A. (2009). Deciphering the immune function and regulation by a TLR of the cytokine EMAPII in the lesioned central nervous system using a leech model. J. Immunol. 183, 7119-7128.

Shimazu, R., Akashi, S., Ogata, H. Nagai, Y., Fukudome, K., Miyake, K. and Kimoto, M. (1999). MD-2, a molecule that confers lipopolysaccharide responsiveness on Tolllike receptor 4. J. Exp. Med. 189, 1777-1782.

Takano, T., Kondo, H., Hirono, I. Endo, M., Saito-Taki, T., and Aoki, T. (2007). Molecular cloning and characterization of Toll-like receptor 9 in Japanese flounder, Paralichthys olivaceus. Mol. Immunol 44, 1845-1853.

Takeda, K., and Akira, S. (2005). Tolllike receptors in innate immunity. Int. Immunol. 17, 1-14.

Wu, B., Xin, B., Jin, M., Wei, T. and Bai, Z. (2011). Comparative and phylogenetic analyses of three TIR domain-containing adaptors in metazoans: implications for evolution of TLR signaling pathways. Dev. Comp. Immunol. 35, 764-773.

Wu, H., Wang, H., Xiong, W., Chen, S., Tang, H., and Han, D. (2008). Expression patterns and functions of toll-like receptors in mouse sertoli cells. Endocrinology 149, 4402-4412.
Yu, Y., Zhong, Q., Li, C., Jiang, L., Yan, F., Wang, Z., and Zhang, Q. (2009). Isolation and characterization of Toll-like receptor 9 in half-smooth tongue sole Cynoglossus semilaevis. Fish Shellfish Immunol. 26, 492-499. Yuan, S., Huang, S., Zhang, W., Wu, T. Dong, M., Yu, Y., Liu, T., Wu, K., Liu, H., Yang, M., Zhang, H., and $\mathrm{Xu}$, A. (2009). An amphioxus TLR with dynamic embryonic expression pattern responses to pathogens and activates NF- $\mathrm{B}$ pathway via MyD88. Mol. Immunol. 46, 2348-2356.

Conflict of Interest Statement: The authors declare that the research was conducted in the absence of any commercial or financial relationships that could be construed as a potential conflict of interest.

Received: 28 November 2011; accepted: 15 February 2012; published online: 29 February 2012.

Citation: Satake $H$ and Sekiguchi $T$ (2012) Toll-like receptors of deuterostome invertebrates. Front. Immun. 3:34. doi: 10.3389/fimmu.2012.00034

This article was submitted to Frontiers in Molecular Innate Immunity, a specialty of Frontiers in Immunology.

Copyright () 2012 Satake and Sekiguchi. This is an open-access article distributed under the terms of the Creative Commons Attribution Non Commercial License, which permits non-commercial use, distribution, and reproduction in other forums, provided the original authors and source are credited. 